\title{
IDH Family Mutation Analysis Was Not Performed
}

National Cancer Institute

\section{Source}

National Cancer Institute. IDH Family Mutation Analysis Was Not Performed. NCI

Thesaurus. Code C160380.

An indication that IDH family mutation analysis was not performed during the study. 